\title{
Genetic Variability and Correlation Analysis for Agronomic and Fibre Quality Traits in Intraspecific Cotton (G. hirsutum $\times$ G. hirsutum) Recombinant Inbred Lines (RILs)
}

\author{
Mahesh R. Hampannavar ${ }^{1 *}$, B. R. Patil ${ }^{1}$, I. S. Katageri ${ }^{1}$, \\ B. N. Aravinda Kumar ${ }^{2}$ and B. S. Janagoudar ${ }^{3}$ \\ ${ }^{1}$ Department of Genetics and Plant Breeding, College of Agriculture, University of \\ agricultural sciences, Dharwad Karnataka-580005, India \\ ${ }^{2}$ Department of Agronomy, College of Agriculture, University of agricultural sciences, \\ Dharwad Karnataka-580005, India \\ ${ }^{3}$ Department of Crop Physiology, College of Agriculture, University of agricultural sciences, \\ Dharwad Karnataka-580005, India \\ *Corresponding author
}

\section{A B S T R A C T}

Keywords

Cotton,

Recombinant inbred lines (RIL), phenotypic (PCV) and genotypic (GCV) coefficient of variation

Article Info

Accepted:

15 December 2019 Available Online:

20 January 2020
There is a limited genetic variability available in genetic resources of upland cotton (Gossypium hirsutum L.), which restricted the breeding activity. The objective of present study was evaluating the genetic variability in RIL population derived through intraspecific hybridization between RHAP 12 and RHAP 15 genotypes. The 222 RIL were evaluated during kharif 2018 at University of Agricultural Sciences Dharwad. The RILs were evaluated for agronomic and fibre quality traits such as plant height $(\mathrm{cm})$, number of monopodial branches, number of sympodia branches, boll per plant, seed cotton yield per plant $(\mathrm{g})$, seed Index $(\mathrm{g})$, boll weight $(\mathrm{g})$, lint index $(\mathrm{g})$, ginning out turn $(\%)$, fiber strength $(\mathrm{g} / \mathrm{text})$, fiber length $(\mathrm{mm})$, micronaire value $(\mu \mathrm{g} / \mathrm{inch})$, maturity coefficient $(\%)$, fiber elongation $(\%)$ and fiber uniformity ratio $(\%)$. The RIL were exhibited the large variation for seed cotton yield per plant i.e. PCV of 34.44 per cent and GCV of 28.05 per cent. Similarly number of bolls per plant also exhibited the high genetic variation with PCV and GCV of 28.26 and 21.34 per cent respectively. Even though after utilizing diverse parents in hybridization program observed the limited variability with respect to fibre quality traits. The high heritability and genetic advance over the mean registered in seed cotton yield per plant indicating that additive gene action. The desirable association with yield was found between number of bolls per plant, number of sympodia per plant, boll weight and maturity ratio. It is concluded that RIL population had high potential for enhancing the yield and fibre quality traits. 


\section{Introduction}

Cotton is the corner stone in textile industry and known as white gold. Out of 50 species of gossypium only four species have been cultivated for fiber viz., Gossypium arboretum, Gossypium herbaceum, Gossypium hirsutum and Gossypium barbadense (Brubaker et al., 1999). India is the only country in the world where all four species can be grown. The species Gossypium hirsutum L. have been growing in larger proportion because higher yield potential and broader adaptability. In cotton, improving the fibre yield and quality are two main objectives. With this thrust, breeders must always develop new elite cultivars with both high yield and improved quality. The success of any breeding programme depends on the range of genetic variability present in the population. A wider spectrum of variability will improve the chances of selecting elite genotype. Some researchers opinioned that intensive selection imposed to maximize yield and adaptation, along with selection for early maturity, has eliminated substantial variation from elite $G$. hirsutum germplasm pools. So it is necessary to expand the genetic resource in cotton through various means viz, recombination, genetic transformation and mutation etc. Therefore, the present study was undertaken to find out the genetic variability, heritability and genetic advance of yield and its component and fibre quality parameters in 222 Recombinant Inbred Lines (RILs) derived from a cross between $G$. hirsutum var. RHAP 12 and G. hirsutum var. RHAP 15.

Seed cotton yield is the complex character which is product of interaction of many characters. Grafius (1959) opinioned that "there may not be any gene for yield as such, but operates only through its components". Its essential to know the component characters involved in interaction and way there association with yield parameter. Association study provides the better understanding of yield attributes, which assists the plant breeder during the selection (Robinson et al., 1951 and Johnson et al., 1955). In this study association between the various quantitative were also studied.

\section{Materials and Methods}

The present study comprised of 222 recombinant inbred lines (RILs) of $\mathrm{F}_{6}$ generation were produced by intraspecific crossing between RHAP 12 and RHAP 15 lines of G. hirsutum L. The RILs and their parent with one check variety (MCU 5) were evaluated in simple lattice design with two replications at agricultural sciences Dharwad, during kharif 2018. Each replication consisted of 225 genotypes. Each line was grown with spacing of $90 \mathrm{~cm}$ between rows and $30 \mathrm{~cm}$ between plants respectively under deep black cotton soil. The randomly selected five plants from each line were used for observing the following traits $v i z$, plant height $(\mathrm{cm})$, number of monopodial branches, number of sympodia branches, boll per plant, seed cotton yield per plant (g), seed Index (g), boll weight (g), lint index $(\mathrm{g})$, ginning out turn (\%), fiber strength (g/text), fiber length $(\mathrm{mm})$, micronaire value $(\mu \mathrm{g} / \mathrm{inch})$, maturity coefficient (\%), fiber elongation (\%), fiber uniformity ratio (\%).

The analysis of variance (Panse and Sukhatme, 1985) was estimated using SAS 9.4 software (SAS, 2004). The genotypic coefficient of variance (GCV) and phenotypic coefficient of variance (PCV) were calculated by formula given by Burton (1952), heritability (\%) in broad sense was worked out by using formula suggested by Lush (1949) and genetic advance as per cent of mean were estimated by using the procedure given by Lush (1949) and Johnson et al., (1955a). The genotypic and phenotypic correlations estimated by using procedure suggested by Johnson et al., (1955b). 


\section{Results and Discussion}

All the recombinant inbred lines were displayed considerable amount of differences in their mean performance with respect to all the characters considered for study. The mean sum square due to genotypes showed the significance difference for all characters, suggesting that the derived lines were genetically variable and considerable amount of variability existed among them (Table 1). The mean, range, PCV, GCV, heritability and genetic advance over the mean were calculated for above mention quantitative traits in RIL population (Table 2). The RIL population had high variability for seed cotton yield per plant, number of bolls per plant and boll weight. It indicated that selection was effective in the RIL population with considering these three traits. All the fibre quality traits had low PCV and GCV except micronaire value. The RIL population had less variability considering the fibre quality traits since RIL population was derived from the biparental hybridization and intraspecific hybridization. The high heritability $\left(\mathrm{h}^{2}\right)$ and genetic advance over the mean (GAM) was observed in traits such as seed cotton yield per plant, boll weight, number of bolls per plant and micronaire value which indicated that these traits were governed by additive gene action. The similar results were reported by Choukdi et al., (2012), Hafiz et al., (2013), Dhivya et al., (2014), Ahsan et al., (2015), Fakhar et al., (2015), Muhammad et al., (2015), Eswari et al., (2016), Nagaraju (2016) and Ramesh et al., (2018).

Seed cotton yield in cotton is the complex character which is product of interaction of many characters. The seed cotton yield in cotton is dependent and low heritable trait so selection of genotypes by considering the independent traits which had the significant association with yield will simplify the breeder work. Its necessary to know the component characters involved in interaction and way there association with yield parameter. Association study provides the better understanding of yield attributes which assists the plant breeder during the selection (Robinson et al., 1951 and Johnson et al., 1955b). Considering this discussion the traits like plant height, number of monopodia per plant, number of bolls per plant, boll weight, number of sympodia per plant, ginning outturn and lint index were exhibited a significant positive correlation with seed cotton yield per plant (Table 3). While at genotypic level an additional trait i.e. seed index had significant positive correlation along listed traits (Table 4). In previous reports by Farooq et al., (2014), Ramesh (2015) Gopi and Patil (2017), Kumar (2017), Raza et al., (2016), Nikhil et al., (2018) were reported the same result. While considering the fibre quality characteristics, the fibre strength and fibre length could improve simultaneously because both had a significant positive correlation (Ramesh, 2015; Malagouda et al., 2016 and Kumar, 2017). The seed cotton yield had non significant negative correlation with fibre strength and fibre length (Neelam and Potdukhe, 2002; Altaher and Singh (2003).

The fibre maturity ratio and micronaire value had significant association with yield among fibre quality traits at genotypic level however at phonotypic value only fibre maturity ratio had significant association with yield (Neelima, 2002 and Altaher and Singh, 2003).

In case of correlation we measure the degree and direction of association two characters while path analysis estimates the cause of association between the two characters. The idea of path analysis was initially developed by Wright in 1921, but this analysis first used in plant breeding for selection by Dewey and Lu (1959). 
Table.1 Analysis of variance for yield and its component and fibre quality traits in the RIL population of G. hirsutum

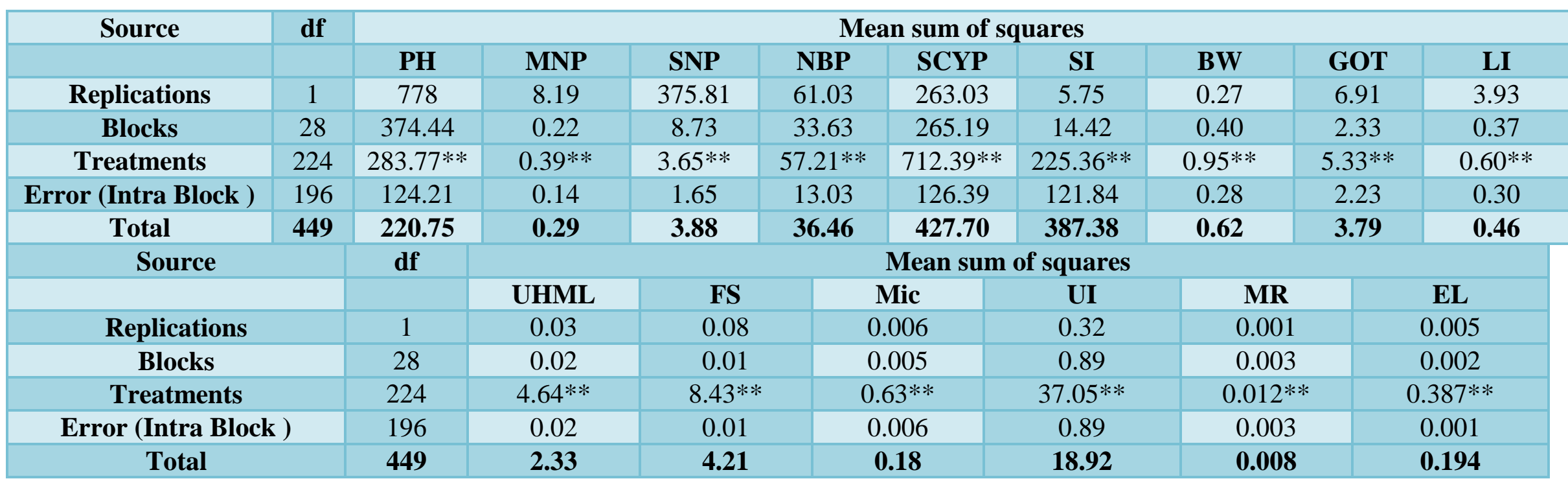

Where,

$\mathrm{PH}=$ Plant height $(\mathrm{cm}), \mathrm{MNP}=$ Number of monopodia per plant, $\mathrm{SNP}=$ Number of sympodia per plants, NBP = Number of bolls per plant, $\mathrm{SCYP}=\mathrm{Seed}$ cotton yield per plant $(\mathrm{g}), \mathrm{SI}=$ Seed index $(\mathrm{g}), \mathrm{BW}=$ Boll weight $(\mathrm{g})$, GOT $=$ Ginning outturn $(\%)$, LI = Lint index (g), FS = Fibre strength $(\mathrm{g} / \mathrm{tex})$, UHML = Upper half mean length $(\mathrm{mm}), \quad$ Mic $=$ Micronaire value $(\mu \mathrm{g} / \mathrm{inch}), \mathrm{UI}=$ Uniformity index $(\%), \mathrm{MR}=$ Maturity ratio, FEL $=$ Fibre elongation length $(\%)$, $* *-$ Significance at 5 per cent. 
Table.2 Mean, range and component of variance of yield and its component traits and fibre quality traits in recombinant inbred lines of $G$. hirsutum

\begin{tabular}{|c|c|c|c|c|c|c|c|c|}
\hline $\begin{array}{l}\text { Sl. } \\
\text { No. }\end{array}$ & Characters & Mean \pm SE & Range Lowest & $\begin{array}{c}\text { Range } \\
\text { Highest }\end{array}$ & PCV & GCV & $\begin{array}{c}h^{2} \text { (Broad } \\
\text { Sense) }\end{array}$ & $\begin{array}{c}\text { Gen. Adv as } \\
\% \text { of Mean } \\
(5 \%)\end{array}$ \\
\hline $\mathbf{1}$ & $\mathrm{PH}$ & $116.59 \pm 8.87$ & 69.88 & 148.40 & 12.76 & 6.85 & 28.00 & 7.57 \\
\hline 2 & MNP & $2.40 \pm 0.27$ & 1.30 & 3.80 & 21.65 & 14.27 & 43.50 & 19.38 \\
\hline 3 & SNP & $9.76 \pm 1.12$ & 5.90 & 14.50 & 17.97 & 7.48 & 17.30 & 6.41 \\
\hline 4 & NBP & $21.46 \pm 2.81$ & 10.90 & 41.50 & 28.26 & 21.34 & 57.00 & 33.18 \\
\hline 5 & SCYP & $60.37 \pm 8.53$ & 15.70 & 135.00 & 34.44 & 28.05 & 66.30 & 47.04 \\
\hline 6 & SI & $6.54 \pm 0.55$ & 4.88 & 8.90 & 13.80 & 6.82 & 24.40 & 6.95 \\
\hline 7 & BWP & $2.85 \pm 0.39$ & 1.26 & 4.92 & 27.89 & 19.97 & 51.30 & 29.47 \\
\hline 8 & GOT & $39.26 \pm 1.06$ & 35.39 & 42.68 & 4.96 & 3.13 & 40.00 & 4.08 \\
\hline 9 & LI & $4.24 \pm 0.39$ & 2.88 & 6.51 & 16.06 & 8.99 & 31.40 & 10.38 \\
\hline 10 & FS & $25.27 \pm 0.06$ & 22.33 & 30.63 & 8.04 & 8.03 & 99.80 & 16.53 \\
\hline 11 & UHML & $24.94 \pm 0.11$ & 20.40 & 30.90 & 6.03 & 6.00 & 98.90 & 12.29 \\
\hline 12 & Mic & $3.53 \pm 0.05$ & 2.16 & 4.68 & 12.28 & 12.07 & 96.70 & 24.45 \\
\hline 13 & UI & $75.67 \pm 0.66$ & 58.20 & 94.40 & 5.57 & 5.43 & 95.00 & 10.90 \\
\hline 14 & MR & $0.57 \pm 0.04$ & 0.29 & 0.78 & 16.08 & 11.68 & 53.70 & 17.46 \\
\hline 15 & FEL & $5.03 \pm 0.03$ & 4.00 & 6.60 & 8.66 & 8.60 & 98.60 & 17.60 \\
\hline
\end{tabular}

Where, $\mathrm{PH}=$ plant height $(\mathrm{cm}), \mathrm{MNP}=$ number of monopodia per plant, $\mathrm{SNP}=$ number of sympodia per plants, $\mathrm{NBP}=$ Number of bolls per plant, $\mathrm{SCYP}=$ seed cotton yield per plant (g), SI= seed index (g), BW = Boll weight (g), GOT = Ginning outturn (\%), LI = Lint index (g), FS = Fibre strength (g/tex), UHML = Upper half mean length $(\mathrm{mm})$, Mic $=$ Micronaire value $(\mu \mathrm{g} / \mathrm{inch}), \mathrm{UI}=$ Uniformity index $(\%), \mathrm{MR}=$ Maturity ratio, FEL $=$ Fibre elongation length $(\%)$. PCV phenotypic coefficient of variation, GCV = genotypic coefficient of variation. 
Table.3 Phenotypic correlation coefficient between seed cotton yield and its component traits and fibre quality traits in 222 recombinant inbred lines of $G$. hirsutum

\begin{tabular}{|c|c|c|c|c|c|c|c|c|c|c|c|c|c|c|c|}
\hline $\begin{array}{c}\text { Characte } \\
\text { rs }\end{array}$ & PH & MNP & SNP & NBP & SI & BW & GOT & LI & UHML & FS & Mic & UI & MR & EL & SCYP \\
\hline PH & 1.000 & 0.042 & 0.090 & -0.011 & 0.069 & $0.295 * *$ & -0.048 & 0.041 & -0.014 & -0.028 & $0.167 * *$ & -0.080 & 0.076 & $-0.173 * *$ & $0.216^{* *}$ \\
\hline MNP & & 1.000 & 0.069 & $0.209 * *$ & 0.073 & -0.009 & 0.033 & 0.082 & -0.009 & -0.022 & $0.127 * *$ & $0.096^{*}$ & $0.098^{*}$ & -0.046 & $0.174 * *$ \\
\hline SNP & & & 1.000 & $0.373^{* *}$ & 0.077 & -0.029 & 0.000 & 0.074 & -0.0003 & 0.026 & 0.062 & 0.109 & 0.052 & $0.250 * *$ & $0.294 * *$ \\
\hline NBP & & & & 1.000 & -0.051 & $-0.168 * *$ & $0.102 *$ & 0.015 & -0.045 & -0.028 & 0.064 & $0.202 * *$ & 0.041 & $0.243 * *$ & $0.672 * *$ \\
\hline SI & & & & & 1.000 & $0.156^{* *}$ & -0.004 & $0.860 * *$ & 0.081 & $0.108 *$ & $0.126^{* *}$ & -0.048 & 0.082 & 0.032 & 0.085 \\
\hline BW & & & & & & 1.000 & $0.108 *$ & $0.186 * *$ & -0.009 & -0.059 & $0.256^{* *}$ & $-0.112 *$ & $0.164 * *$ & $-0.225^{* *}$ & $0.593 * *$ \\
\hline GOT & & & & & & & 1.000 & $0.501 * *$ & -0.029 & 0.019 & $0.173 * *$ & 0.0008 & $0.123 * *$ & 0.018 & $0.206 * *$ \\
\hline $\mathbf{L I}$ & & & & & & & & 1.000 & 0.052 & $0.100 *$ & $0.196^{* *}$ & -0.041 & $0.131 * *$ & 0.032 & $0.182 * *$ \\
\hline UHML & & & & & & & & & 1.000 & $0.640 * *$ & $-0.176^{* *}$ & $0.115^{*}$ & $-0.098 *$ & $0.159 * *$ & -0.065 \\
\hline FS & & & & & & & & & & 1.000 & $-0.164 * *$ & $0.182 * *$ & $-0.131 * *$ & $0.258 * *$ & -0.027 \\
\hline Mic & & & & & & & & & & & 1.000 & -0.079 & $0.859 * *$ & $-0.196 * *$ & -0.066 \\
\hline UI & & & & & & & & & & & & 1.000 & 0.036 & $0.435 * *$ & 0.060 \\
\hline MR & & & & & & & & & & & & & 1.000 & $-0.125^{* *}$ & $0.154 * *$ \\
\hline EL & & & & & & & & & & & & & & 1.000 & 0.007 \\
\hline SCYP & & & & & & & & & & & & & & & 1.000 \\
\hline
\end{tabular}

Where,

$*$ = significance at $5 \%$ level

$* *$ = significance at $1 \%$ level

$\mathrm{PH}=$ plant height $(\mathrm{cm}), \mathrm{MNP}=$ number of monopodia per plant, $\mathrm{SNP}=$ number of sympodia per plants, NBP $=\mathrm{Number}$ of bolls per plant, $\mathrm{SCYP}=$ seed cotton yield per plant $(\mathrm{g}), \mathrm{SI}=$ seed index $(\mathrm{g}), \mathrm{BW}=$ Boll weight $(\mathrm{g}), \mathrm{GOT}=$ Ginning outturn $(\%), \mathrm{LI}=\mathrm{Lint}$ index $(\mathrm{g}), \mathrm{FS}=$ Fibre strength $(\mathrm{g} / \mathrm{tex}), \mathrm{UHML}=\mathrm{Upper}$ half mean length $(\mathrm{mm})$, Mic $=$ Micronaire value $(\mu \mathrm{g} / \mathrm{inch}), \mathrm{FUI}=$ Fibre uniformity index $(\%), \mathrm{MR}=$ Maturity ratio, FEL $=$ Fibre elongation length $(\%)$. 
Table.4 Genotypic correlation coefficient between seed cotton yield and its component traits and fibre quality traits in 222 recombinant inbred lines (RILs) of G. hirsutum

\begin{tabular}{|c|c|c|c|c|c|c|c|c|c|c|c|c|c|c|c|}
\hline $\begin{array}{c}\text { Char } \\
\text { acter } \\
\text { S }\end{array}$ & PH & MNP & SNP & NBP & SI & BW & GOT & LI & UHML & FS & Mic & UI & MR & EL & SCYP \\
\hline PH & $\begin{array}{c}1.00 \\
0\end{array}$ & $\begin{array}{c}0.129 * \\
*\end{array}$ & $\begin{array}{c}0.224 * \\
*\end{array}$ & $-0.205 * *$ & $0.315 * *$ & $0.554 * *$ & $0.146 * *$ & $\begin{array}{c}0.338 * \\
*\end{array}$ & -0.003 & -0.043 & $0.358 * *$ & $-0.103^{*}$ & $0.410 * *$ & $-0.300 * *$ & $0.249 * *$ \\
\hline MNP & & 1.000 & 0.010 & $0.211^{* *}$ & $0.141^{* *}$ & 0.083 & 0.091 & $\begin{array}{c}0.172 * \\
*\end{array}$ & -0.012 & -0.042 & $0.198 * *$ & $0.153 * *$ & $0.219 * *$ & -0.067 & $0.198 * *$ \\
\hline SNP & & & 1.000 & $0.987 * *$ & 0.034 & $-0.224 * *$ & $-0.151 * *$ & -0.044 & -0.009 & 0.060 & $0.137 * *$ & $0.249 * *$ & $0.098^{*}$ & $0.593 * *$ & $0.648^{* *}$ \\
\hline NBP & & & & 1.000 & -0.064 & 0.027 & $0.232 * *$ & 0.081 & -0.056 & -0.036 & $0.092 *$ & $0.281 * *$ & $0.100 *$ & $0.325^{* *}$ & $0.759 * *$ \\
\hline SI & & & & & 1.000 & $0.250 * *$ & 0.081 & $\begin{array}{c}0.825^{*} \\
*\end{array}$ & $\begin{array}{c}0.160 * \\
*\end{array}$ & $\begin{array}{c}0.216^{*} \\
*\end{array}$ & $0.248 * *$ & $-0.113^{*}$ & $0.173 * *$ & 0.049 & $0.119 * *$ \\
\hline BW & & & & & & 1.000 & $0.167 * *$ & $\begin{array}{c}0.285^{*} \\
*\end{array}$ & -0.015 & -0.083 & $0.359 * *$ & $\begin{array}{c}- \\
0.167 * *\end{array}$ & $0.290 * *$ & $-0.321 * *$ & $0.662 * *$ \\
\hline GOT & & & & & & & 1.000 & $\begin{array}{c}0.628 * \\
*\end{array}$ & -0.040 & 0.032 & $0.287 * *$ & 0.013 & $0.317 * *$ & 0.027 & $0.329 * *$ \\
\hline LI & & & & & & & & 1.000 & 0.092 & $\begin{array}{c}0.179 * \\
*\end{array}$ & $0.355 * *$ & -0.076 & $0.135^{* *}$ & 0.046 & $0.278 * *$ \\
\hline $\begin{array}{c}\text { UHM } \\
\mathbf{L}\end{array}$ & & & & & & & & & 1.000 & $\begin{array}{c}0.639 * \\
*\end{array}$ & $\begin{array}{c}- \\
0.200 * *\end{array}$ & $0.094 *$ & $-0.236^{* *}$ & $0.151 * *$ & -0.031 \\
\hline FS & & & & & & & & & & 1.000 & $\begin{array}{c}- \\
0.176^{* *}\end{array}$ & $0.176^{* *}$ & $-0.225^{* *}$ & $0.256^{* *}$ & -0.080 \\
\hline Mic & & & & & & & & & & & 1.000 & $\begin{array}{c}- \\
0.125^{* *}\end{array}$ & $0.988 * *$ & $-0.219 * *$ & $0.308 * *$ \\
\hline UI & & & & & & & & & & & & 1.000 & $-0.167 *$ & $0.427 * *$ & 0.080 \\
\hline MR & & & & & & & & & & & & & 1.000 & $-0.264 * *$ & $0.278 * *$ \\
\hline EL & & & & & & & & & & & & & & 1.000 & 0.009 \\
\hline $\begin{array}{c}\text { SCY } \\
\mathbf{P}\end{array}$ & & & & & & & & & & & & & & & 1.000 \\
\hline
\end{tabular}

Where,

$*$ = significance at $5 \%$ level

$* *=$ significance at $1 \%$ level

$\mathrm{PH}=$ plant height $(\mathrm{cm}), \mathrm{MNP}=$ number of monopodia per plant, $\mathrm{SNP}=$ number of sympodia per plants, NBP = Number of bolls per plant, $\mathrm{SCYP}=$ seed cotton yield per plant $(\mathrm{g})$, SI= seed index (g), BW = Boll weight $(\mathrm{g})$, GOT = Ginning outturn (\%), LI = Lint index (g), FS = Fibre strength (g/tex), UHML = Upper half mean length $(\mathrm{mm})$, Mic $=$ Micronaire value $(\mu \mathrm{g} / \mathrm{inch}), \mathrm{FUI}=$ Fibre uniformity index $(\%), \mathrm{MR}=$ Maturity ratio, FEL $=$ Fibre elongation length $(\%)$. 
Table.5 Phenotypic path coefficient among seed cotton yield and its attributing and fibre quality traits in 222 recombinant inbred lines of G. hirsutum

\begin{tabular}{|c|c|c|c|c|c|c|c|c|c|c|c|c|c|c|c|}
\hline Characters & PH & MNP & SNP & NBP & SI & BW & GOT & LI & UHML & FS & Mic & UI & MR & EL & SCYP \\
\hline PH & 0.009 & 0.000 & 0.001 & 0.000 & 0.001 & 0.003 & 0.000 & 0.000 & 0.000 & 0.000 & 0.002 & -0.001 & 0.001 & -0.002 & $0.216^{* *}$ \\
\hline MNP & 0.001 & 0.013 & 0.001 & 0.003 & 0.001 & 0.000 & 0.000 & 0.001 & 0.000 & 0.000 & 0.002 & 0.001 & 0.001 & -0.001 & $0.174^{* *}$ \\
\hline SNP & 0.002 & 0.002 & 0.027 & 0.010 & 0.002 & -0.001 & 0.000 & 0.002 & 0.000 & 0.001 & 0.002 & 0.003 & 0.001 & 0.007 & $0.294 * *$ \\
\hline NBP & -0.009 & 0.163 & 0.292 & 0.782 & -0.040 & -0.131 & 0.080 & 0.012 & -0.035 & -0.022 & 0.051 & 0.159 & 0.033 & 0.190 & $0.672 * *$ \\
\hline SI & -0.013 & -0.014 & -0.015 & 0.010 & -0.189 & -0.030 & 0.001 & -0.163 & -0.016 & -0.021 & -0.024 & 0.009 & -0.015 & -0.006 & 0.085 \\
\hline BW & 0.209 & -0.006 & & -0.119 & & & 0.077 & & -0.006 & -0.042 & 0.183 & -0.080 & 0.117 & -0.160 & \\
\hline GOT & 0.003 & -0.002 & 0.000 & -0.007 & 0.000 & -0.007 & -0.064 & -0.032 & 0.002 & -0.001 & -0.011 & 0.000 & -0.008 & -0.001 & $0.206^{* *}$ \\
\hline LI & 0.010 & 0.019 & 0.017 & 0.003 & 0.199 & 0.043 & 0.116 & 0.231 & 0.012 & 0.023 & 0.046 & -0.009 & 0.030 & 0.007 & $0.182 * *$ \\
\hline UHML & 0.000 & 0.000 & 0.000 & -0.001 & 0.003 & 0.000 & -0.001 & 0.002 & 0.030 & 0.020 & -0.005 & 0.004 & -0.003 & 0.005 & -0.027 \\
\hline FS & 0.001 & 0.000 & 0.000 & 0.000 & -0.002 & 0.001 & 0.000 & -0.002 & -0.010 & -0.016 & 0.003 & -0.003 & 0.002 & -0.004 & -0.066 \\
\hline Mic & -0.001 & -0.001 & 0.000 & 0.000 & -0.001 & -0.001 & -0.001 & -0.001 & 0.001 & 0.001 & -0.005 & 0.000 & & 0.001 & $0.244^{* *}$ \\
\hline UI & 0.001 & -0.001 & -0.001 & -0.003 & 0.001 & 0.001 & 0.000 & 0.001 & -0.001 & -0.002 & 0.001 & -0.012 & 0.000 & -0.005 & 0.06 \\
\hline MR & 0.000 & 0.000 & 0.000 & 0.000 & & 0.000 & & 0.000 & & & -0.002 & 0.000 & -0.003 & 0.000 & $0.154 * *$ \\
\hline EL & 0.004 & 0.001 & -0.006 & -0.006 & -0.001 & 0.005 & 0.000 & -0.001 & -0.004 & -0.006 & 0.005 & -0.010 & 0.003 & -0.024 & 0.008 \\
\hline
\end{tabular}

Residual effect $=\mathbf{0 . 1 7 5}$

Where,

$*=$ significance at $5 \%$ level, $* *=$ significance at $1 \%$ level

$\mathrm{PH}=$ plant height $(\mathrm{cm}), \mathrm{MNP}=$ number of monopodia per plant, $\mathrm{SNP}=$ number of sympodia per plants, NBP $=\mathrm{Number}$ of bolls per plant, $\mathrm{SCYP}=$ seed cotton yield per plant (g), SI= seed index (g), BW = Boll weight $(\mathrm{g})$, GOT = Ginning outturn (\%), LI = Lint index (g), FS = Fibre strength $(\mathrm{g} / \mathrm{tex})$, UHML = Upper half mean length $(\mathrm{mm})$, Mic $=$ Micronaire value $(\mu \mathrm{g} / \mathrm{inch}), \mathrm{FUI}=$ Fibre uniformity index $(\%), \mathrm{MR}=$ Maturity ratio, FEL = Fibre elongation length $(\%)$. 
Fig.1 Path analysis of seed cotton yield per plant in 222 recombinant inbred lines derived from RHAP 12 and RHAP 15 genotypes



The path analysis was calculated at phenotypic and genotypic level but genotypic values of some the traits were very negligible hence here discussed path analysis only at phenotypic level (Table 5 and Fig. 1). At phenotypic level three traits like number of bolls per plant, boll weight and lint index had direct positive effect on seed cotton yield per plant hence direct selection through these trait will be helpful. All the observed fibre quality traits had low or negligible direct effect on yield so in such situation; the multi trait selection was recommended. In this particular investigation residual effect was low i.e. 0.175. which indicates the other possible independent characters which were not included in the study.

From the results, it can be concluded that phenotypic selection in the RIL population would be more effective for improvement seed cotton yield per plant, number of bolls per plant and boll weight because these characters had high GCV, PCV. heritability and GAM. Both at phenotypic and genotypic level number of bolls per plant, number of sympodia per plant, boll weight, maturity ratio had significant positive correlation with seed cotton yield. The number of bolls per plant and boll weight had the high direct and indirect effect on yield in cotton RIL population.

\section{References}

Ahsan, M. Z., Majidano, M. S., Bhutto, H., Soomro, A. W., Panhwar, F. H., Channa, A. R., and Sial, K. B., 2015. Genetic variability, coefficient of variance, heritability and genetic 
advance of some Gossypium hirsutum L. accessions. J. Agric. Sci. 7(2): 147151.

Brubaker, C. L., Paterson, A. H., and Wendel, J. F., 1999. Comparative genetic mapping of allotetraploid cotton and its diploid progenitors. Genome 42:184-203

Burton, G.W., 1952. Quantitative inheritance in grasses. Proceedings of 6th International Grassland Congress, 1, 277-283.

Choudki, V. M., Katageri, I. S., Patil, R. S., and Vamadevaiah, H. M., 2012. Evaluation of $\mathrm{F}_{11}$ recombinant lines of $G$. herbaceum $\times G$. anomalum and $G$. herbaceum $\times G$. barbadense crosses for fibre traits in cotton. Karnataka $J$. Agric. Sci. 25(4): 414-417.

Dhivya, R. P., Amalabalu, R. P., and Kavithamani, D., 2014. Variability, heritability and genetic advance in upland cotton (Gossypium hirsutum L.). African J. Plant Sci. 8(1): 1-5.

Eswari, K. B., Sudheer, K., Gopinath, S., and Rao, M. V. B., 2016. Genetic variability heritability and genetic advance studies in cotton. Int. J. Dev. Res. 3: 1-3.

Fakhar, Z. K., Shoaib, U. R., Muhammad, A. A., Waqas, M., Chaudhry, M. H., Muhammad, B., Ghulam, Q., Asif, L., Javaria, A., and Umar, F., 2015, Exploitation of germplasm for plant yield improvement in cotton (Gossypium hirsutum L.). J. Green Physiol. Genet. Genomics. 11: 1-10.

Grafius, J. E., 1959, Heterosis in barley. Agron. J. 51: 551-554.

Hafiz, G. A., Abid, M., and Qurban, A., 2013. Genetic variability, heritability, genetic advance and correlation studies in cotton (Gossypium hirsutum L.). Int. Res. J. Microbiol. 4(6): 156-161.

Johnson, H. W., Robinson, H. F. and Comstock, R. E., 1955a, Estimates of genetic and environmental variability in soybean. Agron. J., 47: 314-318.

Johnson, H. W., Robinson, H. F., and Comstock, R. E., 1955b. Genotypic and phenotypic correlations in soybean and their implications in selection. Agron. J. 47: 477-483.

Lush, J. L., 1949. Heritability, genetic advance and character association on rabi sorghum. Hereditas. 2: 356-375.

Muhammad, Z. A., Muhammad, S. M., Hidayatullah, B., Abdul, W. S., Hussain, F. P., Abdul, R. C., and Karim, B. S., 2015. Genetic variability, coefficient of variance, heritability and genetic advance of some Gossypium hirsutum L. accessions. J. Agril. Sci. 7(2): 38-42.

Nagaraju, C. H., 2016. Studies on genetics of QTLs for yield, yield component and fibre quality traits in cotton. Ph.D. Thesis, University Agricultural Scinces, Dharwad, Karnataka, India.

Panse, V. G., and Sukhatme, P. V., 1967. Statistical Methods for Agricultural Workers, 2nd Edition, Indian Council of Agricultural Research, New Delhi.

Panse, V. G., 1940. Application of genetics to plant breeding II. The inheritance of quantitative characters and plant breeding. J. Genet. 40: 283-302.

Ramesh, U. M., Katageri, I. S., and Mohan Kumar, N. V., 2018. Genetic variability and transgressive segregation studies in inter specific cotton (Gossypium hirsutum $\times G$. barbadense) recombinant inbred lines population. J. Farm Sci. 31(4): 373 377.

Robinson, H. F., Comstock, R. E., and Harvey, P. H., 1951. Genotypic and phenotypic correlations in corn and their implications in selection. Agron. J. 43: 262-267.

Farooq, J., Anwar, M., Riaz, M., Farooq, A., Mahmood, A., Shahid, M. T. H., 
Rafiq, M., and Ilahi, F., 2014. Correlation and path coefficient analysis of earliness, fibre quality and yield contributing traits in cotton (Gossypium hirsutum L.). J. Anim. Plant Sci. 24(3): 781-790.

Ramesh, 2015. Genome mapping and identification of molecular markers for various agronomic and fibre quality traits in tetraploid cotton. Ph. D. Thesis, University Agricultural sciences, Dharwad, Karnataka (India).

Gopi, T. J. S., and Patil, B. R., 2017. Genetic variability, correlation and path analysis in $\mathrm{F}_{2}$ generation of interspecific cross of Gossypium arboreum and Gossypium herbaceum for yield and its component traits. Int. J. Pure Appl. Biosci. 5(6): 300-306.

Kumar, M. N. V., and Katageri, I. S., 2017. Genetic variability and heritability study in $\mathrm{F}_{2}$ population of Gossypium barbadense L. cotton for yield and its components. Int. J. Curr. Microbiol. Appl. Sci. 6 (6): 975-983.

Raza, H., Khan, N. U., Khan, S. A., Gul, S., Latif, A., Hussain, I., Khan, J., Raza, S., and Baloch, M., 2016. Genetic variability and correlation studies in $\mathrm{F}_{4}$ populations of upland cotton. J. Anim. Plant Sci. 26(4): 1048-1055.

Nikhil, P. G., Nidagundi, J. M., and Anusha, H. A., 2018. Correlation and path analysis studies of yield and fibre quality traits in cotton (Gossypium hirsutum L.). J. Pharmacognosy Phytochem. 7(5): 2596-2599.

Malagouda, P., Khadi, B. M., Basamma, K., and Katageri, I. S., 2014. Genetic variability and correlation analysis for fibre quality traits in diploid cotton (Gossypium spp.). American Eurasian J. Agric. Environ. Sci. 14(5): 392-395.

Neelam, G. D., and Potdukhe, N. R., 2002. Studies on variability and correlations in upland cotton for yield and its components. J. Indian Soc. cotton Improv. 27(3): 148-152.

Altaher, A. F., and Singh, R. P., 2003. Genetic diversity studies in upland cotton (Gossypium hirsutum L.). J. Indian Soc. cotton improv. 28(3): 158-163.

Wright, S., 1921. Correlation and causation. J. Agric. Res. 20: 202-209.

Dewey, D. R., and Lu, K. H., 1959. Correlation and path analysis of component of created wheat grass seed production. Agron. J. 51: 513-518.

\section{How to cite this article:}

Mahesh R. Hampannavar, B. R. Patil, I. S. Katageri, B. N. Aravinda Kumar and Janagoudar, B. S. 2020. Genetic Variability and Correlation Analysis for Agronomic and Fibre Quality Traits in Intraspecific Cotton ( $G$. hirsutum $\times G$. hirsutum) Recombinant Inbred Lines (RILs). Int.J.Curr.Microbiol.App.Sci. 9(01): 493-503. doi: https://doi.org/10.20546/ijcmas.2020.901.054 